\title{
Fünfzig Jahre Schielbehandlung - Ein Erfahrungsbericht aus Klinik und Praxis
}

\author{
Elfriede Stangler-Zuschrott
}

Eingegangen: 13. Mai 2020 / Angenommen: 8. Januar 2021 / Online publiziert: 28. Januar 2021

(C) Der/die Autor(en) 2021

\begin{abstract}
Zusammenfassung Der Aufbau der orthoptischen Abteilung an der historischen I. Univ.-Augenklinik Wien zwischen 1960 und 1997 und die internationalen Fortschritte werden beschrieben, jedoch auch die besonderen Behandlungsmethoden der Klinik, wie z. B. der Ausgleich des Schielwinkels mit Prismen vor Schieloperationen oder die Prismenbehandlung von kindlichem Strabismus ohne Operation. Bei Entlassung von der Klinik fühlt sich der Patient als subjektiv geheilt. Die Nachhaltigkeit der Therapie zeigt sich erst später in der augenärztlichen Praxis. Die Vielzahl von Amblyopien, konsekutiven Divergenzen, Diplopien oder Horror fusionis nach mehrfachen Schieloperationen lässt unsere Schielbehandlung als fragwürdig erscheinen. Schielen ist nur selten ein anatomisches Problem, vielmehr ein funktionelles. Ohne Fusion ist ein dauerhafter Parallelstand der Augen nicht möglich. Daher ist für die Zukunft ein geändertes Programm zu empfehlen: weniger Operationen in der Kindheit, mehr Fusionstraining mit Prismen und dem jungen Patienten mehr Zeit geben für seine Entwicklung.
\end{abstract}

Schlüsselwörter Strabismus .

Fusionstraining · Langzeitprismenbehandlung · Amblyopiebehandlung · Folgen mehrfacher Schieloperationen · Alignment

Prof. Dr. E. Stangler-Zuschrott ( $\bowtie)$

Universitätsklinik für Ophthalmologie und Optometrie

Wien, emer., Landstraßer Hauptstraße 141/11, 1030 Wien, Österreich

e.stangler-zuschrott@aon.at
Fifty years of strabismology-A report of clinical and practical experiences

Summary The development of the orthoptic department at the famous I. University Eye Clinic in Vienna between 1960-1997 and the international progress of science are described as well as the specific treatment methods of this clinic, such as prism treatment prior to squint surgery or prism treatment without any surgery at all. The patients are discharged from the clinic thinking that they have been cured; however, the aftereffects are first seen later in the ophthalmological practice. The multitude of patients with amblyopia, diplopia and horror fusionis after multiple squint operations seem to make our treatment of strabismus questionable. A squint position commonly arises not due to a dysfunction of an ocular muscle but due to absent or insufficient fusion power. Therefore, in future we should promote a new program: less operations in infancy, prolonged fusion training with the help of prisms and the young child should be given more time for development.

Keywords Strabismus · Fusion training · Long-term prism treatment - Amblyopia treatment - Sequelae of multiple squint operations $\cdot$ Alignment

\section{Problemstellung}

Von Kollegen wird die Autorin manchmal gefragt: Wie hat man um 1960 den Strabismus behandelt, und welche Fortschritte hat die Strabologie im Laufe der Jahre erzielt? Diese Fragestellung ist sehr komplex und nur subjektiv zu beantworten, da manche Themen trotz jahrelanger Kontroversen immer noch umstritten sind. Dieser Bericht bezieht sich daher nur auf die Behandlungsmethoden der historischen I. Univ.Augenklinik in Wien, „Sehschule“, deren Leitung die 
Autorin von 1967 bis 1997 innehatte. Danach folgten weitere 17 Jahre Erfahrung in der Ordination und mehr Zeit für eigene Publikationen. Der Sinn dieses Berichts liegt in einer Weitergabe bewährter Methoden der Schielbehandlung v. a. an praktizierende Kollegen, wobei die Forschungsergebnisse der I. Augenklinik Wien eingeflossen sind. Auf die umfangreiche Literatur dieses Zeitraums kann nur in Einzelfällen hingewiesen werden.

\section{Rückblick. Der Beginn 1960-1967}

Unter der Klinikleitung von Prof. Arnold Pillat hatte der 1. Oberarzt Udo Nemetz alle orthoptisch-pleoptischen Geräte beschafft, die international üblich waren, jedoch heute kaum noch jemand kennt: Pleoptophor, Euthyskop, Lokalisator, Separator, Cheiroskop, Tiefentrainer, Lesepult und mehrere Synoptophore. Es fand sich auch eines der ersten Polatest-Geräte, damals von der Fa. Busch erzeugt.

In der Sehschule arbeiteten 2 Ärzte in Rotation und 2 instruierte Hilfsschwestern, ausgebildete Orthoptistinnen gab es nicht. Erste Lehrer der Strabologie waren F.A. Hamburger und Hans Rotter.

Der Ablauf der Schielbehandlung entsprach im Wesentlichen der heute noch üblichen:

1. Brillenbestimmung nach Zylinderskiaskopie in Atropin- oder Homatropin-Mydriase, wobei die gefundenen hyperopen Werte um eine halbe Dioptrie reduziert verordnet wurden. Die Brillenverordnung erfolgte allerdings erst im 3. Lebensjahr.

2. Die Diagnostik des Strabismus beruhte auf: Abdecktest, Fixationsprüfung, Synoptophorbefund (3 Grade des Binokularsehens nach Worth) und dem Worth-four-dot-Test.

3. Die Amblyopiebehandlung erfolgte im 3. bis 4 . Lebensjahr zunächst mit Atropinkur des führenden Auges, ab 4 Jahren mit inverser Okklusion über 6 bis 10 Wochen bei exzentrischer Fixation, danach mit Okklusionsverbänden oder Schielklappen des führenden Auges ganztägig ohne Unterbrechung und nach Altersschema: 2 Jahre - 1 Woche lang, 3 Jahre -2 Wochen lang etc., wissend, dass $\mathrm{zu}$ langes Abdecken iatrogen eine Schädigung des führenden Auges bewirken kann, welche in späteren Jahren als Deprivationsamblyopie bezeichnet werden sollte. Sobald ein Visus von 6/18 erreicht war, wurde mit einer Binokularschulung am Synoptophor begonnen, wodurch sich die Schielstellung oft reduzierte. Seit 1955, wohl unter dem Einfluss von St. Gallen, wurde bei exzentrischer Fixation die direkte Okklusion als kontraindiziert angesehen und Pleoptik nach Bangerter oder Cüppers durchgeführt. Die Erfolge waren gut bei anfänglichem Vorliegen von zentraler Fixation.

4. Die Binokularschulung erfolgte ausschließlich am Synoptophor. Vorbereitend wurde über mehrere Wochen alternierende Okklusion am Brillenglas durchgeführt, um die Entwicklung einer anomalen Netzhautkorrespondenz (abgekürzt als ANK im deutschen, ARC im angloamerikanischen Sprachraum) zu verhindern bzw. zu unterbrechen. Laut Bericht von Nemetz [1] gelang es in vielen Fällen, NRC („normal retinal correspondence“) wieder zu aktivieren, als Voraussetzung für die Heilung des Strabismus. Dies steht im Gegensatz zur Meinung von Lang [2], ARC könne nicht geheilt werden. Anders und positiv zu bewerten bis in die heutige Zeit sind Fusionsübungen, nicht nur für Schielende, sondern v. a. bei asthenopischen Beschwerden.

5. An Schieloperationen wurden durchgeführt: Tenotomien v. a. am Rectus internus, Resektionen des Externus und Myektomien des Obliquus inferior. Nach der Operation gab es Blickverbände, um das Ergebnis zu verbessern. Leider hatten wir Jahre später große Schwierigkeiten, konsekutive Divergenzen $\mathrm{zu}$ revidieren, da ein tenotomierter Muskel atrophiert.

6. Kontrollbehandlungen wurden bis zum 15. Lebensjahr durchgeführt.

\section{Der Aufenthalt in St. Gallen (Schweiz)}

In den Jahren 1965 bis 1967 waren insgesamt 10 Monate verteilter Gastarzttätigkeit an der Augenklinik St. Gallen möglich sowie an der angeschlossenen OPOS (Ostschweizerische Pleoptik-Orthoptik-Station) unter der Gesamtleitung von Prof. Bangerter, der ein hervorragender Operateur war. Die Leitung der OPOS hatte er an Prof. Joachim Otto übergeben. Die ärztliche Belegschaft bestand aus Schweizern (u.a. Balder Gloor vor seinem Wechsel in die USA), Deutschen und Österreichern in bestem Einvernehmen. Als Wienerin fiel der Autorin die Aufgabe zu, die Zylinderskiaskopie nach Lindner mit den Kollegen zu üben. Es gab Gesprächsrunden und kleine Symposien, an denen Prof. Roland Brückner und seltener auch Josef Lang teilnahmen.

Ein Kollege von St. Gallen, Dr. Graemiger, besprach mit uns die Operationsindikation der operativen Fälle. Eine persönliche Assistentin von Prof. Otto war die Dipl.-Orthoptistin Sr. Gisela Rabetge, deren feine Beobachtungsgabe vorbildlich war und deren Künste am Synoptophor in einem Buch festgehalten sind [3].

Gespräche mit Prof Otto ergaben grundlegende neue Einsichten zum Verständnis des Schielens, die weitgehend mit den Auffassungen von F.A. Hamburger übereinstimmten:

Dominanz und Wettstreit sind prinzipielle Verhaltensweisen Schielender, gleichgültig, ob amblyop oder nicht. Dominanztypen zeigen oft geringe Anisometropien, tendieren zu Suppression, sind aber fusionswillig. Wettstreittypen sind fusionsunwillig, tendieren zum Alternieren und reagieren auf bifoveales Bildangebot mit Ausweichbewegungen. Diese Tatsache erschwert die Anpassung von Prismen und macht die Ergebnisse von Operationen oft unberechenbar. 
Methoden und Resultate der klinischen orthoptisch-pleoptischen Abteilung nach 1967

\section{Der Beginn an der Klinik}

Das Interesse der Kliniker an Orthoptik war nicht groß. Zwei erfahrene Hilfsschwestern unterstützten die Autorin als neue Leiterin der Sehschule, die alle Untersuchungen und Behandlungen selbst durchführte, jedoch nur selten operieren durfte (anfangs noch Tenotomien). Dienstposten für diplomierte Orthoptistinnen mussten erst geschaffen werden. In Salzburg gab es bereits eine Schule für den orthoptischen Dienst, eine Absolventin des ersten Jahrgangs kam 1969 nach Wien und blieb bis zu ihrer Pensionierung. Schließlich teilte die Klinikleitung der Sehschule einen Ausbildungsassistenten auf jeweils ein halbes Jahr zu, um wichtige Kenntnisse wie Schielbehandlung, Skiaskopie und Brillenverordnung zu erlernen. Damit gab es einen Assistenten für Schieloperationen, deren $\mathrm{Zu}$ ständigkeit nun ganz auf die Sehschule überging.

Eine weitere Erschwernis waren fehlende Lehrbücher. Nach dem veralteten Buch von Lyle-Jackson [4] und dem vergriffenen Beiheft von Hollwich [5] war die einzige „Bibel“ für Strabologie das Buch von Krüger [6]. Erst 1971 erschien die erste Auflage von J. Lang „Strabismus, Diagnostik, Schielformen, Therapie“ und 1974 jene des Meisterwerkes von Burian und v. Noorden „Binocular Vision and Ocular Motility“.

Langs Buch ist bis heute für den Unterricht unerreicht, weil es mit nötiger Präzision in Kürze Schielformen beschreibt, die es Arzt bzw. Orthoptist(in) erlaubt, die Symptomatik eines Patienten in die richtige Schublade einzuordnen mit entsprechenden therapeutischen Konsequenzen.

Befruchtend für alle Strabologen von Wien und Umgebung waren die monatlichen Gesprächsrunden um F.A. Hamburger, die seit ca. 1960 in der Bibliothek der I. Augenklinik stattfanden. Hamburger war Mitglied des international besetzten Consilium Europaeum Strabismi Studio deditum, sinngemäß nannte sich unsere Interessensgemeinschaft bei unserem ersten Symposium 1967 dann Consilium Strabologicum Austriacum.

\section{Die Arbeitsweise der neuen Sehschule}

1. Diagnostik Vom Synoptophor weg wurde jetzt im freien Raum untersucht: Schielwinkelmessung mit Prismenabdecktest in variablen Distanzen; die Anschaffung einer Tangententafel nach Harms sowie eines Lees-Schirms anstelle des alten Hess-Schirms zur Messung von Inkomitanzen; und schließlich erhielten wir ein Phasendifferenzhaploskop nach Aulhorn, das viele Möglichkeiten an Diagnostik und Therapie eröffnete. Die einfachste und genialste Erfindung aber war jene der Streifengläser von Bagolini [7], die es erlauben, das Binokularsehen nahezu ohne Dissoziation zu prüfen.
2. Brillenverordnung Grundprinzip für Schielende ist bis heute die Verordnung der Vollkorrektur des Refraktionsfehlers jeglicher Art nach Skiaskopie unter Atropin oder Cyclopentolat, wobei Atropin um $+0,5 \mathrm{dpt}$ höhere Werte aufdeckt. Die Unterkorrektur einer Hyperopie bei Divergenz ist wegen des meist gleichzeitig vorhandenen Fehlers der Akkommodation nicht zu empfehlen. Andererseits führt eine von Réthy (1967) vorgeschlagene Überkorrektur einer Hyperopie nicht zu einer stärkeren Reduktion eines konvergenten Schielwinkels als die Vollkorrektur. Ein Verdienst Réthys liegt in der dringlichen Aufforderung zur Frühbehandlung schielender Kinder, denen er schon im Babyalter Brillen mit binasaler Okklusion verordnete, nach dem Motto „Vertane Zeit erschwert den Erfolg“ (Phaedrus).

3. Amblyopiebehandlung Trotz seiner Ablehnung der Okklusion bei exzentrischer Fixation war Bangerter immer der Meinung, Prophylaxe sei die beste Therapie; deshalb schickte er seine Orthoptistinnen in die Kindergärten, um Schielfälle rechtzeitig aufzudecken. In Österreich sieht der 1974 eingeführte Mutter-Kind-Pass eine augenärztliche Untersuchung von Kindern im Alter von 24 bis 30 Monaten vor, worauf die Inzidenz schwerer Amblyopien wesentlich seltener wurde.

Aufgrund der revolutionären Forschungen von Hubel und Wiesel und v. Noorden ergab sich eine neue Einteilung der Amblyopien: a) Deprivationsamblyopien als Folge optischer Hindernisse sind meist nicht heilbar. b) Anisometropische Amblyopien, bei ihnen genügt der Refraktionsausgleich, nach einiger Zeit stellt sich spontan verbesserter Visus ein, zusätzliche Teilzeitokklusion ist ein Vorteil. c) Die Schielamblyopie ist bis zum Ende des 3. Lebensjahres durch Okklusion fast immer reversibel, wo nicht, ist ein organischer Fehler zu vermuten, der nicht immer lokalisierbar ist. Vor Schuleintritt und danach werden die Heilungschancen immer geringer.

Unser Behandlungsschema für Kinder von 5 bis 12 Jahren in Kürze: bei unsteter Fixation Beginn mit Ansteigeokklusion nach Lang wegen der raschen Ermüdbarkeit und psychischen Belastung der Kinder. Bei exzentrischer Fixation trägt man inverse Okklusion auf 4 Wochen (zu lange Dauer bewirkt im amblyopen Auge zusätzliche Deprivation!); bei Lockerung der Fixation Versuch mit direkter Okklusion mit tageweisem Wechsel je nach Alter. War bei Misserfolg eine Übungstherapie in der Sehschule nötig, bestand diese aus mehreren Stufen:

a. Bewegungsübungen des amblyopen Auges (Gerät egal, viele wurden versucht) zur Auflockerung der Fixation,

b. optische Stimulation der Makula (z.B. Pleoptophor, Lichtstreifenreizung [8], Konvergenzspirale, Schachbrettmuster von VEP-Geräten, CAM-VisionTrainer, diverse elektronische Programme etc.), 
c. Lokalisationsübungen mit einfachen Mitteln oder Koordinator nach Cüppers,

d. Sehübungen mit bewegten Objekten (z. B. EinzelOptotypen-Methode nach Rabetge).

Damit erreichten wir bei dem genannten Krankengut zu $36 \%$ einen bleibenden Visus von 0,8 bei entsprechender Nachbehandlung. Bessere Ergebnisse (59\% Normalisierung) erreicht man hingegen bei Mikrostrabismen. Diese sind allerdings ein Problem. Von Kollegen wurden wir vor dem Auftreten unkorrigierbarer Doppelbilder nach Amblyopiebehandlung gewarnt. Zum Glück hatten wir nie einen derartigen Fall. Eine Erklärung dafür? Mikrotropien sind mit Fusionsfähigkeit begünstigt. Diese Fusion wie auch die Suppressionsfähigkeit sollten erhalten bleiben; daher keine inverse Okklusion und zur Nachbehandlung nur Sichtfolien von maximal 0,1 für das führende $\mathrm{Au}-$ ge; außerdem ist ein Visus von 0,8 ein ausreichender Erfolg.

Viele Ideen und Vorschläge zur Amblyopiebehandlung wurden im Lauf der Jahre entwickelt. Etwa die von Pfandl angedachte, von Pouliquen und Quéré propagierte Penalisation entspricht einem Fern-Nah-Alternans zur Behandlung leichterer Amblyopien mit zentraler Fixation. In Wien wurde sie wegen des „Giftes Atropin“ von vielen Eltern nicht angenommen, auch erschien uns eine Sicht- oder Teilzeitokklusion effektiver. Die moderne elektronische Überwachung der Okklusionszeiten ist gewiss ein Fortschritt. Hingegen konnten neuere Computerspiele wie etwa das „Binocular iPad Game“ nicht überzeugen. Der Stein der Weisen ist immer noch die Prophylaxe.

4. Fusionsschulung Versuche zur Änderung der Netzhautkorrespondenz werden nicht mehr unternommen. Positiv zu bewerten aber bis in die heutige Zeit sind Fusionsübungen; vor und nach Operationen, nicht für Schielende allein, sondern v. a. bei asthenopischen Beschwerden, denen oft eine Fusionsstörung zugrunde liegt. Dazu ein guter Rat von F.A. Hamburger: Fusionsübungen sollten am Synoptophor mit großen Bildern zur Stimulation der peripheren Fusion begonnen werden, um erfolgreich $\mathrm{zu}$ sein, die erweiterten peripheren Panumareale lassen dies $\mathrm{zu}$, auch bei der harmonischer ARC des Mikrostrabismus. In Kombination mit Konvergenztraining, Übungen mit Prismenleisten oder am Aulhorn-Gerät sind sie ohne Alterslimit auch bei Senioren mindestens für die Dauer eines halben Jahres effektiv.

\section{Operationen sowie Vor- und Nachbehandlung}

Die freie Tenotomie wurde verlassen, ebenso die routinemäßige Rücklagerung beider Interni gleichzeitig wegen der Gefahr späterer Divergenzen. Standardoperation war bei Konvergenz und Divergenz die kombinierte Operation (Rücklagerung+Resektion). Häufigstes Operationsalter das Ende des 3. Lebensjahrs. Die Fadenoperation diente (wie ursprünglich von Cüppers 1971 vorgesehen) als Gegenparese zu einer Parese und nicht als Routineoperation etwa bei schwankendem Schielwinkel. Bei schweren Abduzensparesen wurde die Jensen-Operation durchgeführt. Die Obliquuschirurgie wurde verfeinert, justierbare Nähte selten angewandt und Botulinumtoxin nur bei Blepharospasmus.

Eine Enttäuschung ist es für jeden Operateur $\mathrm{zu}$ sehen, wenn kunstvoll parallel gestellte Augen bereits nach 1 Monat wieder schielen. Die fehlende „Harmonie im gleichzeitigen Sehen“ [9], zu verstehen als periphere Fusion, müsste bereits vor der Operation geübt werden. Dies wollten wir mit ständig, etwa 1 Jahr lang getragenen Prismen bei Strabismus convergens versuchen. Erste Ergebnisse [10] waren ermutigend: Bereits die ersten 48 Fälle zeigten gegenüber den herkömmlich behandelten Patienten: postoperatives Alignment (=Winkel von $\pm 2^{\circ}$ ) um $24 \%$ verbessert, neuerlicher Winkelanstieg postoperativ um $50 \%$ vermindert und die Zahl der geheilten Fälle von $13 \%$ auf $39 \%$ angestiegen.

Eine spätere statistisch überprüfte Arbeit [11] ergab Ähnliches: a) Verbesserung der Operationsergebnisse von früher $68 \%$ auf $93 \%$; b) um $50 \%$ geringerer Anstieg des Schielwinkels im ersten Jahr postoperativ; c) weniger Zweitoperationen desselben Patienten $(8 \%$ statt früher 25,6\%); d) Verdoppelung der Zahl der auch sensorisch geheilten Fälle sowie die Entwicklung von peripherer Fusion selbst beim frühkindlichen Schielsyndrom.

Die Nachbehandlung erfolgte, falls nötig, wieder mit Prismen oder anderen orthoptischen Möglichkeiten.

6. Schielbehandlung ohne Operation Mit Ausnahme einiger Schielformen akkommodativer Art galt seit Jahrzehnten das Prinzip, Schielen könne nur mithilfe des Messers geheilt werden. Folgeerscheinungen von Operationen in der Kindheit zeigen sich oft viele Jahre später, am häufigsten mit beginnender Presbyopie. Dies fordert die ärztliche Verantwortung heraus, nicht $\mathrm{zu}$ früh und zu oft $\mathrm{zu}$ operieren oder auch überhaupt nicht. Unsere erste Studie [12] von konservativ mit Prismen behandelten 44 Patienten, Alter 2 bis 14 Jahre (d. 3,4), zeigte Folgendes: Zunächst nimmt der Schielwinkel zu - eine bekannte Tatsache -, nach längstens 6 Monaten tritt Stabilität ein [13], nach einer variierenden Dauer ständig geübter sensorischer Orthophorie vermindert sich der anatomische Schielwinkel oft innerhalb weniger Monate, und die Prismen werden abgebaut. Die Behandlungsdauer bei Strabismus convergens betrug 2 bis 6,5 Jahre (d. 3,4 Jahre), jüngere Kinder sprachen besser an. Ein Versagen mussten wir nur bei Ausgangsschielwinkeln größer als $+15^{\circ}$ feststellen. Die Erfolgsrate betrug 29/37 (78\%), 6 Fälle waren Heilungen nach Lang [2], der Rest kosmetisch unauffällige Mikrotropien.

Eine spätere Arbeit befasste sich mit intermittierendem Strabismus divergens und Prismentherapie [14]; 
19 Patienten erreichten Parallelstand ohne Operation, 20 weiteren dienten die Prismen zur Vor- und Nachbehandlung einer Operation. Zuletzt hatten alle Patienten eine gut kompensierte Exophorie, Heilungen sind selten. Ein Schielwinkel größer als $-10^{\circ}$ erfordert eine Operation. Die Behandlungsdauer ist bei reiner Prismentherapie (d. 4,25 Jahre) länger als in Kombination mit einer Operation (d. 3,45 Jahre). Im Vergleich zu konvergentem Schielen ist der Divergens schwieriger und langwieriger $\mathrm{zu}$ behandeln.

In jedem Fall ist die Angst vor einer Amblyopie durch Prismenfolien unbegründet, wenn diese auf beide Augen verteilt werden, prismatische Brillengläser zusätzlich verwendet werden und man darauf achtet, dass sich kein Mikrostrabismus hinter der Brille entwickelt.

\section{Geriatrische Orthoptik als neues Wissensgebiet}

Die erste Hilfesuchende kam Ende 1970 in die Sehschule, war vergeblich bei mehreren Augenärzten und Neurologen gewesen; die zweite war eine praktische Ärztin, die über Zeitungsinserat ihr Leid klagte. Bald führte ein kleineres Kollektiv zur ersten Publikation des Zustandsbildes: „Strabismus convergens des Presbyopenalters" [15], Charakteristika: über lange Zeit subjektiv nicht realisierte ungekreuzte Doppelbilder auf Distanz, Lesen ungestört. Jahre später nannte de Decker dies „seniles Schielen“ [16], in Amerika wurde es als Divergenzparese eingestuft. Seit Mittelman [17] heißt diese Krankheit nun international ,age-related distance esotropia“. Die Therapie ist einfach: Prismen oder eine Operation.

An Senioren waren vor 1980 Schieloperationen verpönt gewesen wegen der Gefahr von Diplopie. Unsere Publikation [18] war die erste auf diesem Gebiet. Seitdem steigt die Zahl derartiger Operationen.

Alterserkrankungen der Augen sind v. a. Katarakt, Glaukom und Makulopathie. Besonders bei letzterer Erkrankung wurden von uns etliche Studien der Binokularfunktionen durchgeführt, die in einem kleinen Buch zusammengefasst sind [19].

8. Binokulare simultane Infrarotokulographie zur Registrierung feinster Augenbewegungen Nach langer Suche gelang es in Zusammenarbeit mit Ing. Didier Bouis ein routinemäßig einsetzbares, sehr genaues Gerät ähnlich einem Synoptophor herzustellen [20]. Damit konnten viele neue Studien durchgeführt und Erkenntnisse gewonnen werden, am wichtigsten vielleicht das Auftreten anomaler Bewegungsmuster nach visueller Deprivation Erwachsener oder nach Schädel-Hirn-Traumen [20, 21]. Diese Arbeiten stellen eine Ergänzung zur Monographie „Horror fusionis“ (1970) von F.A. Hamburger dar.

\section{Die Resultate aus der Ordination}

Sie zeigen die langfristigen Ergebnisse der von der Klinik entlassenen Patienten auf.
Hier finden sich unter 1012 binokular gestörten $\mathrm{Pa}-$ tienten 120 Fälle konsekutiven Schielens, Alter 23 bis 70 Jahre, die in der Kindheit mehrfach operiert worden waren. Davon haben 53 Patienten Diplopie (43\%), von ihnen 8 einen Horror fusionis, 70 sind amblyop (58\%), und nur $30 \%$ haben eine kosmetisch unauffällige Augenstellung. Keine gute Bilanz.

\section{Schlussfolgerungen}

Während der letzten 50 Jahre hat sich die Behandlung schielender Kleinkinder nicht wesentlich verändert: Brille - Okklusion gegen Amblyopie - Operation. Die Brille hat ihren fixen Platz als erste wichtige Maßnahme.

Zur Frage der Okklusion. Die früher geübte Wechselokklusion zur Prophylaxe der ARC ist längst verlassen, seit man weiß, dass die kortikalen binokularen Neurone einen Input brauchen. Auch zur Amblyopiebehandlung sollte man nicht länger als nötig mit Pflastern abdecken, besser Sichtfolien verwenden. Die visuelle Deprivation Erwachsener führt zum Verlust der Fusion und damit zu nicht korrigierbaren Doppelbildern; Kinder reagieren vielleicht rascher als Erwachsene mit Verlust der Fusionsfähigkeit auf das Fehlen binokularer Reize, wie es eine überlang angewandte Okklusion darstellt. Unsere Vorgänger in der Sehschule taten wohl das Richtige, zum Amblyopietraining zusätzlich auch Fusionsübungen zu machen. Möglicherweise wären auf diese Art Doppelbilder bei pleoptisch behandelten Mikrostrabismen zu vermeiden. Untersuchungen diesbezüglich sind uns nicht bekannt.

Vor einer Schieloperation an Kindern sollten folgende Fakten beachtet werden:

a. Kinder mit kongenitalem Schielsyndrom vermindern ihren Schielwinkel spontan zwischen 2. und 5. Lebensjahr um durchschnittlich $10^{\circ}$ (A. Salomon); 20 klinische Fälle zeigten noch vor Schuleintritt Alignment ohne Operation, wenn einer der folgenden Faktoren vorlag: Hohe Hypermetropie, Myopie, mentale Retardation.

b. Kriterien, die eine konsekutive Divergenz erwarten lassen, sind konform mit der Literatur: schwere Amblyopie (Visus 0,2 und geringer), hohe Anisometropie (3dpt), hohe Hypermetropie (6dpt).

c. Übermäßige operative Schwächung der Interni führt zu A-Inkomitanzen und mit zunehmendem Alter zu Leseschwierigkeiten. Mit der Zahl der Operationen steigt die Häufigkeit von Spätkomplikationen. Ärztliches Ziel ist es, mit einer Operation das Therapieziel zu erreichen. An unserer Klinik gelang dies zu $70 \%$, wohl durch die Vorbereitung mit Prismen.

Die Prismen sind die derzeit wichtigste Möglichkeit der konservativen Schielbehandlung. Wegen der anfänglichen Schielwinkelzunahme sind sie umstritten und führen bei Erwachsenen oft zur Operation. Anders ist die Sachlage bei Kindern. Donders war der 
Erste, der 1847 bis 1848 ein allmähliches Nachlassen des Schielwinkels durch Prismen unter Einfluss der peripheren Fusion beschrieben hat [22]. Erst die Erfindung der Wafer-Prismen machte ihre Anwendung praktikabel. Behandlungsziele sind Orthophorie, Heterophorie oder Mikrotropie. Diese sind ohne Operation erreichbar, wenn der Schielwinkel bei Konvergenz $+15^{\circ}$ und bei Divergenz $-10^{\circ}$ nicht übersteigt. Unabhängig von der Schielform war die Behandlungsdauer bei Konvergenz 2 bis 6,5 (d 3,4) Jahre, die Erfolgsrate um $80 \%$; bei Divergenz war die Behandlungsdauer 0,5 bis $8,0(\mathrm{~d} 4,3)$ Jahre, die Erfolgsrate $90 \%$.

Das Einsparen von Operationen entspricht gewiss dem hippokratischen Gedanken des „Primum nil nocere“.

Bei dem vorliegenden Beitrag handelt es sich um einen historischen Rückblick auf die Schielbehandlung an einer der beiden Sehschulen des Wiener AKH aus Sicht der Autorin.

Funding Open access funding provided by Medical University of Vienna.

Interessenkonflikt E. Stangler-Zuschrott gibt an, dass kein Interessenkonflikt besteht.

Open Access Dieser Artikel wird unter der Creative Commons Namensnennung 4.0 International Lizenz veröffentlicht, welche die Nutzung, Vervielfältigung, Bearbeitung, Verbreitung und Wiedergabe in jeglichem Medium und Format erlaubt, sofern Sie den/die ursprünglichen Autor(en) und die Quelle ordnungsgemäß nennen, einen Link zur Creative Commons Lizenz beifügen und angeben, ob Änderungen vorgenommen wurden.

Die in diesem Artikel enthaltenen Bilder und sonstiges Drittmaterial unterliegen ebenfalls der genannten Creative Commons Lizenz, sofern sich aus der Abbildungslegende nichts anderes ergibt. Sofern das betreffende Material nicht unter der genannten Creative Commons Lizenz steht und die betreffende Handlung nicht nach gesetzlichen Vorschriften erlaubt ist, ist für die oben aufgeführten Weiterverwendungen des Materials die Einwilligung des jeweiligen Rechteinhabers einzuholen.

Weitere Details zur Lizenz entnehmen Sie bitte der Lizenzinformation auf http://creativecommons.org/licenses/by/4. 0/deed.de.

\section{Literatur}

1. Nemetz UR, Pressina M, Schmidt I. Amblyopie- und Schielbehandlung an der I. Univ.-Augenklinik in Wien. Klin MonatsblAugenheilkd. 1954;124:266-77.

2. LangJ. WelcheSchielfällekönnen geheilt werden? Ophthalmologica. 1968;156:190-6.

3. OttoJ.Lehrbuchund Atlas der Orthoptik. Bern:Huber; 1975. ISBN3-456-80133-5.
4. Lyle,Jackson.PraktischeOrthoptik. München, Berlin, Wien: Urban \& Schwarzenberg; 1957.

5. Hollwich F. Schielen, Pleoptik, Orthoptik, Operation. Bücherei des Augenarztes, Beiheft der Klin. Mbl.Augenheilkd., 38.Heft. 1961.

6. Krüger KE, Tost M, Ullrich C. Physiologische und methodische Grundlagen der Pleoptik und Orthoptik. Abhandlungen aus dem Gebiete der Augenheilkunde, Bd. 14. Leipzig: Thieme; 1964.

7. Bagolini B, Capobianco NM. Subjective space in Comitant squint. Am J Ophthalmol. 1965;59:430-42.

8. Stangler E. Beobachtungen bei der Amblyopiebehandlung mit bewegtem Lichtstreifen. Klin Monatsbl Augenheilkd. 1971;158:67-71.

9. Ritterich F. Lehre von den blutigen Augenoperationen am menschlichen Körper. Leipzig und Heidelberg: C. F. WinterscheVerlagshandlung; 1858.

10. Stangler-Zuschrott E. Prismen als Operationsvorbereitung beim Strabismus convergens alternans. Klin Monatsbl Augenheilkd. 1974;165:909-14.

11. Zehetmayer M, Stangler-Zuschrott E, Schemper M. Prolonged preoperative prismatic treatment in anternating convergent squint. Acta Ophthalmol. 1994;72:103-9.

12. Stangler-ZuschrottE. Long-termwearing of prisms-aconservative way to cure squint deviations in childhood? In: Lennerstrand G, Hrsg. Advances in strabismology, Proceedings of the VIII. meeting of ISA in Maastricht 1998. Buren: Aeolus; 1998. S. 85-8.

13. Stangler-ZuschrottE. Prismen zur Behandlung des Strabismus bei Kindern. Spektrum Augenheilkd. 2012;26:230-5. https://doi.org/10.1007/s00717-012-0120-y.

14. Stangler-ZuschrottE. The treatment of intermittent Exotropia in childhood: a long-term study. In: Mills SL, Hrsg. Eye movement disorders. New York: Nova Verlag; 1916. S. 65-82. ISBN 978-1-63321-980-9.

15. Stangler-Zuschrott E. Strabismus convergens des Presbyopenalters. Klin Monatsbl Augenheilkd. 1976;168:775-83.

16. Dannheim-de Decker E, de Decker W. Strabismus convergensim Senium. ZPrakt Augenheilkd. 1994;16:263-6.

17. Mittelman D. Age-related distance esotropia. J AAPOS. 2006;10:212-2013.

18. Stangler-Zuschrott E. Schieloperationen an presbopen Patienten. Klin MonatsblAugenheilkd. 1981;181:397-9.

19. Stangler-Zuschrott E. Geriatric orthoptics and non-paretic diplopiain adults. New York: Nova SciencePublishers;2016. ISBN 978-1-63485-244-9.

20. Stangler-Zuschrott E, Bouis D. Ein neues binokulares Okulometer zur simultanen Registrierung feinster Augenbewegungen. Spektrum Augenheilkd. 1987;1:113-6.

21. Stangler-Zuschrott E. Horror fusionis. Eine Störung sensomotorischer Funktionen. Spektrum Augenheilkd. 2005;19:280-9.

22. Remky H. Strabismology from its Beginnings. In: v. Noorden GK, Hrsg. The History of Strabismology. Oostende, Belgium:J.P.Wayenborgh;2002.S.35.

Hinweis des Verlags Der Verlag bleibt in Hinblick auf geografische Zuordnungen und Gebietsbezeichnungen in veröffentlichten Karten und Institutsadressen neutral. 